\title{
Consensus Guidelines for the Diagnosis and Treatment of Adults with Growth Hormone Deficiency: Summary Statement of the Growth Hormone Research Society Workshop on Adult Growth Hormone Deficiency*
}

Based on the increasing body of evidence that adults with GH deficiency (somatotropin deficiency) have impaired health that improves with GH replacement, many countries have already approved the use of GH for replacement therapy in adults with GH deficiency. To ensure that patients are appropriately identified and treated, the Growth Hormone Research Society (GRS) convened a workshop on April 1417, 1997, in Port Stephens, Australia, to formulate consensus guidelines for the diagnosis and treatment of adults with $\mathrm{GH}$ deficiency. The GRS invited scientists with expertise in the field, representatives from industry involved in the manufacture of recombinant human $\mathrm{GH}$, and representatives from health authorities from a number of countries to attend the workshop, all of whom contributed to the consensus guidelines as detailed below.

Received July 25, 1997. Accepted October 10, 1997.

Address all correspondence and requests for reprints to: GRS Secretariat, Medical Department M, Aarhus Kommunehospital, DK- 8000, Aarhus C, Denmark.

*Participants in the Growth Hormone Research Society Workshop on Adult Growth Hormone Deficiency, Port Stevens, New South Wales, Australia, April 14-18, 1997: Dr. Andrea Attanasio, Lilly Research Center (England); Dr. Kenneth Attie, Genentech, Inc. (USA); Dr. Rob Baxter, Kollings Institute of Medical Research (Australia); Dr. Bengt-Ake Bengtsson, Sahlgrenska University Hospital (Sweden); Dr. Allan Black, Therapeutic Goods Administration (Australia); Dr. Sandra Blethen, Genentech, Inc. (USA); Dr. Lena Carlsson, Sahlgrenska University Hospital (Sweden); Dr. Filipe Casaneuva, Santiago de Compostela University (Spain); Dr. John Chipman, Lilly Research Laboratories (USA); Dr. Jens Sandahl Christiansen, Aarhus Kommunehospital (Denmark); Dr. David Clemmons, University of North Carolina (USA); Dr. Ross Cuneo, Princess Alexandra Hospital (Australia); Mr. Dirk De Rijdt, Pharmacia \& Upjohn (Belgium); Dr. Ezio Ghigo, University of Turin (Italy); Dr. Mark Hartman, University of Virginia (USA); Ms. Elizabeth Hernberg-Stahl, Pharmacia \& Upjohn (Sweden); Dr. Raymond Hintz, Stanford University Medical Center (USA); Dr. Ken Ho Garvan, Institute of Medical Research (Australia); Dr. David Hoffman, Garvan Institute of Medical Research (Australia); Dr. Minoru Irie, Toho University (Japan); Dr. Jens Otto Jorgensen, Aarhus Kommunehospital (Denmark); Ms. Anne-Marie Kappelgaard, Novo Nordisk A/S (Denmark); Dr. Zvi Laron, Children's Medical Center of Israel (Israel); Dr. Saul Malozowski, FDA (USA); Dr. David Russell-Jones, St. Thomas' Hospital (England); Dr. Steve Shalet, Christie Hospital (England); Dr. Pierre Sizonenko, University of Geneva (Switzerland); Dr. Peter H. Sonksen, St. Thomas' Hospital (England); Dr. Christian Strasburger, Innenstadt University Hospital (Germany); Dr. K. Takano, Tokyo Women's Medical College (Japan); and Dr. Michael Thorner, University of Virginia Health Sciences Center (USA).

\author{
Diagnosis of GH Deficiency in Adults \\ Definition of adult GH deficiency
}

Severe GH deficiency should be defined biochemically within an appropriate clinical context. In patients with hypothalamic-pituitary disease, the syndrome of adult GH deficiency characteristically presents with alterations in body composition, including reduced lean body mass and bone mineral density and increased fat mass with a preponderance of abdominal adiposity (increased waist circumference). The skin is thin and dry, and sweating is reduced. Muscle strength and exercise performance are reduced. An impaired sense of well-being and other psychological complaints are common. Partial GH deficiency exists, but further research is needed to distinguish it from physiological causes of reduced GH secretion, e.g. aging. Furthermore, the benefits of treatment of partial GH deficiency remain to be established.

\section{Patients who should be tested for adult GH deficiency}

An evaluation for GH deficiency should be considered only in patients with evidence of hypothalamic-pituitary disease, subjects who have received cranial irradiation, or patients with childhood onset of GH deficiency. In patients with organic hypothalamic-pituitary disease, the likelihood of GH deficiency increases with the increasing number of pituitary hormone deficits from approximately $45 \%$ if no other deficits are present to nearly $100 \%$ if three or four pituitary hormone deficiencies are present. It may not be necessary to evaluate patients with pituitary microadenomas for GH deficiency unless other pituitary hormone deficits are present or unless a strong clinical suspicion of GH deficiency exists. Patients with childhood-onset GH deficiency should be retested as adults before committing them to long term $\mathrm{GH}$ replacement.

\section{Biochemical diagnosis of adult GH deficiency}

Dynamic tests of GH secretion. The diagnosis of adult GH deficiency is established by provocative testing of GH secretion. Patients should be receiving stable and adequate hormone replacement for other hormonal deficits before testing. At present, the insulin tolerance test is the diagnostic test of choice. Provided adequate hypoglycemia is achieved, this test distinguishes $\mathrm{GH}$ deficiency from the reduced GH secretion that accompanies normal aging and obesity. The in- 
sulin tolerance test should be performed in experienced endocrine units where the test is performed frequently. The test is contraindicated in patients with electrocardiographic evidence or history of ischemic heart disease or in patients with seizure disorders. Given these precautions, the insulin tolerance test is safe; however, there is less experience in patients over the age of $60 \mathrm{yr}$.

Most normal subjects respond to insulin-induced hypoglycemia with a peak GH concentration of more than $5 \mu \mathrm{g} / \mathrm{L}$. Severe GH deficiency is defined by a peak GH response to hypoglycemia of less than $3 \mu \mathrm{g} / \mathrm{L}$. These cut-off values were defined in GH assays employing polyclonal competitive RIAs. However, GH immunoassay results vary between different methods, and therefore, the cut-off value may need to be adjusted appropriately (see below for standardization of assays).

In patients with contraindications to the insulin tolerance test, alternative provocative tests of $\mathrm{GH}$ secretion must be used with appropriate cut-offs. At present, the combined administration of arginine and GHRH is the most promising alternative. Administration of arginine alone or glucagon alone can be considered, but these tests have less established diagnostic value compared to the insulin tolerance test. Other stimulatory tests may prove to be useful, but require further validation. The present data indicate that the clonidine test is less useful in adults than in children.

Adult patients with hypothalamic-pituitary disease and one or more additional pituitary hormone deficits require only one provocative test of GH secretion for the diagnosis of GH deficiency. Childhood-onset GH deficiency requires reconfirmation in adulthood. To establish the diagnosis of isolated GH deficiency in adults, it is recommended that, in addition, a second biochemical test of GH status be abnormal.

Biochemical markers of GH action. Several biochemical markers of GH action have been studied to determine their diagnostic potential in adult GH deficiency. Serum insulin-like growth factor I (IGF-I) concentrations are only useful when ageadjusted normal ranges are available. In adults, a normal serum IGF-I does not exclude the diagnosis of GH deficiency. A serum IGF-I below the normal range is suggestive of GH deficiency in the absence of other conditions known to lower serum IGF-I levels; for example, malnutrition, hepatic disease, poorly controlled diabetes mellitus, and hypothyroidism. In the presence of multiple (two or more) pituitary hormone deficiencies, a low serum IGF-I level indicates a high probability of GH deficiency. However, it is recommended that the diagnosis of adult GH deficiency be confirmed by a provocative test of GH release.

Measurement of serum IGF-binding protein-3 or acidlabile subunit has to date not proven to offer any advantage over the measurement of serum IGF-I.

\section{Standardization of assays}

GH immunoassay results vary between different assay methods. The above recommendations for cut-off values for the insulin tolerance test are based on results obtained with polyclonal competitive RIAs calibrated against the pituitaryderived preparation International Reference Preparation
(IRP) 80/505 (1 mg = 2.6 U). The GRS advocates future use of the recombinant hGH preparation IRP 88/624 $(1 \mathrm{mg}=3.0$ U). Further comparative studies are necessary at both national and international levels to achieve standardization of GH assays.

The presence of IGF-binding proteins interfere with measurement of serum IGF-I. At present, removal of IGF-binding proteins before immunoassay is essential. However, new IGF-I assays are being developed that may not require extraction procedures. The current international reference standard for IGF-I assays is IRP 87/518. GH and IGF-I results should be expressed in mass units.

\section{Treatment of GH Deficiency in Adults}

\section{Patients to be treated}

All patients with documented severe GH deficiency are eligible for GH replacement. The goal for GH replacement in adults is to correct the abnormalities associated with adult GH deficiency.

\section{Dose selection}

The objective of treatment is to maximize benefit and minimize side-effects. Experience has shown that sensitivity to GH treatment varies considerably between individuals, with elderly individuals being the most sensitive. It is recommended that therapy should start with a low dose (0.15-0.30 mg/day; 0.45-0.90 IU/day). The dosage should be increased gradually on the basis of clinical and biochemical responses and no more frequently than at monthly intervals. The maintenance dose may vary considerably from person to person and seldom exceeds $1.0 \mathrm{mg} /$ day (3 IU/day).

In healthy adults it is known that premenopausal women secrete more GH than age-matched men, and that GH secretion is reduced with advancing age and with obesity. In adults aged 30-50 yr, the reported daily production of GH in women was approximately $0.2 \mathrm{mg} /$ day, whereas the production rate in men was approximately $0.1 \mathrm{mg} /$ day. Clinical experience has demonstrated that the variability in sc absorption and individual responsiveness to GH make dose determination based on body weight or body surface area less helpful than anticipated. Furthermore, some adult patients experience side-effects even with a low dose.

In accordance with the clinical practice of treating $\mathrm{GH}$ deficient children, we recommend that $\mathrm{GH}$ be administered as daily sc self-injections in the evening.

\section{Monitoring treatment efficacy}

A physical examination and a careful clinical history with particular attention to quality of life-related questions are of great value in monitoring treatment. Where appropriate, questioning the patient's partner may also assist with the evaluation.

At present, the best biochemical marker of $\mathrm{GH}$ action is IGF-I in serum. An important use of IGF-I measurements is to avoid overreplacement, and values should be kept in the age-related normal range. IGFBP-3 has been found to be less useful, whereas acid-labile subunit, although promising, needs further validation. In the initial stages of dose titration, 
frequent measurements are required (every 1 or 2 months).

Once a stable dose has been reached, once or twice yearly measurements are sufficient.

Simple anthropometric measures, such as weight and waist circumference, may be supplemented by bioelectrical impedance or dual x-ray absorptiometry; the latter is particularly valuable when bone mineral density is reduced. Lipids should be monitored annually.

\section{Safety issues}

Adult GH deficiency is associated with reduced extracellular fluid volume. Fluid retention during GH treatment is, therefore, to be expected, and the patient should be informed. Edema and occasionally carpal tunnel syndrome may be seen. These symptoms are usually transient and dose dependent, but may require a dose reduction. Mild arthralgias may occur, but are usually self-limiting. Because GH may reduce insulin sensitivity, markers of glycemia should be monitored periodically.

The risk of certain malignancies, in particular colon cancer, is increased in acromegaly. It is, however, inappropriate to extrapolate from the acromegalic data to the GH-replaced adult with hypopituitarism. Nonetheless, current recommendations for cancer prevention and early detection in the general population should be implemented.

Good clinical practice requires regular imaging of residual pituitary tumor; GH replacement does not impose a need for intensifying this follow-up. A baseline scan is recommended before starting treatment.
GH influences the metabolism of many substances, including other hormones and medications; therefore, alterations in the dose requirements of such compounds should be anticipated.

\section{Contraindications}

Absolute contraindications include active malignancy, benign intracranial hypertension, and proliferative or preproliferative diabetic retinopathy. Early pregnancy is not a contraindication, although GH should be discontinued in the second trimester as GH is produced by the placenta.

\section{Long-term care}

Although insufficient information is available at present, GH replacement (as with other hormones) is most likely for life. It is possible that the dose requirement may decrease over time. Replacement therapy in the elderly should be monitored particularly carefully as the patient ages, with special emphasis on dosage and perceived benefit. If any patient perceives no benefit, then a trial of withdrawal should be considered.

It is recommended that patients with hypopituitarism receiving GH replacement should remain under long term surveillance by an endocrinologist with special experience in pituitary disease; this can be undertaken in partnership with an internist or general practitioner.

Patients may need initially to be seen initially by the endocrinologist as often as monthly. Once treatment is stabilized, one or two visits per year will suffice. 